\title{
Assessment of bacterial contamination of mobile phones of dentists and dental hygienists by Illumina MiSeq
}

\author{
So Yeon Lee ${ }^{1,2}$, Si Young Lee ${ }^{1,2 \star}$ \\ ${ }^{1}$ Department of Oral Microbiology, College of Dentistry, Gangneung-Wonju National University, Gangneung, Korea \\ ${ }^{2}$ Research Institute of Oral Science, Gangneung-Wonju National University, Gangneung, Korea
}

In a clinical environment, medical staffs often use their mobile phones with their bare hands, thereby possibly exposing them to bacteria contamination. Cross-contamination could occur, where bacteria may be transferred from the contaminated hands of a healthcare worker to a patient. Some studies have investigated the microbial contamination on mobile phones of healthcare workers. However, in these studies, specific bacteria were identified through general culture methods, and few studies have investigated the contamination on mobile phones of dentists. In this study, Illumina sequencing was used to identify the bacterial diversity present on mobile phones of dentists and dental hygienists. Five dentists and five dental hygienists working in a dental hospital were surveyed. Several bacterial genera were detected on the dentists' mobile phone, such as Pseudomonas spp. (52.52\%), Janthinobacterium spp. (14.21\%), Enterococcus spp. (9.66\%), Stenotrophomonas spp. (5.68\%), Streptococcus spp. (4.29\%), and Acinetobacter spp. (3.15\%). On the dental hygienists' mobile phones, Enterococcus spp. (32.02\%), Pseudomonas spp. (23.76\%), Streptococcus spp. (22.44\%), Lactobacillus spp. (8.77\%), Janthinobacterium spp. (3.54\%), Acinetobacter spp. (1.7\%), and Stenotrophomonas spp. (1.22\%) were detected. In addition, $38.09 \%$ of the genera identified on the dentists' mobile phones and $25.74 \%$ of those on the hygienists' mobile phones contained pathogenic species. Bacteria associated with oral diseases, such as Streptococcus spp., Actinomyces spp., Porphyromonas spp., and Fusobacterium spp., were also detected in both groups. This study demonstrated the high rates of bacterial contamination on mobile phones used by dentists and dental hygienists and showed the possibility of contamination by infectious pathogens.

Key Words: Bacteria, Contamination, Illumina Miseq, Mobile phone

(c) This is an open-access article distributed under the terms of the Creative Commons Attribution Non-Commercial License (http://creativecommons.org/licenses/by-nc/4.0) which permits unrestricted noncommercial use, distribution, and reproduction in any medium, provided the original work is properly cited.

\section{INTRODUCTION}

Mobile phones increase the speed of communication within a hospital and are universally used for efficient health care delivery [1-3]. However, as mobile phones often come in contact with the hands of medical staff in hospitals, they can be contaminated by bacteria. Therefore, there is a possibility of cross contamination. Despite their frequent use, mobile phones are not managed cleanly, and there are no guidelines for controlling bacterial contamination on mobile phones.

Since bacteria can be transferred from the contaminated hands of medical staff to patients, it is believed that nosocomial infections can be caused by extrinsic factors, such

Received January 25, 2019; Revised March 13, 2019; Accepted March 15, 2019

Corresponding author: Si Young Lee, Department of Oral Microbiology, College of Dentistry, and Research Institute of Oral Science, Gangneung-Wonju National University, 7 Jukheon-gil, Gangneung 25457, Korea.

Tel: +82-33-640-2455, Fax: +82-33-642-6410, E-mail: siyoung@gwnu.ac.kr

Copyright $\odot$ 2019, Oral Biology Research Institute 
as contaminated medical equipment and the hands of medical staff [4]. In previous studies, the potentially pathogenic microorganisms present on various objects, such as the hands of medical staff and their mobile phones, have been reported [5-8]. According to one study, $9 \%$ to $25 \%$ of the mobile phones used by medical staff were contaminated with pathogens [1]. To prevent the contamination of these mobile phones, it is necessary to set guidelines for hand hygiene and the disinfection of mobile phones. In addition, the use of mobile phones should be reconsidered in locations where the risk of infection is high, such as operating rooms and intensive care units.

Infectious microorganisms attached to a surface can survive for long periods of time unless they are removed by processes such as disinfection or sterilization [9], and contaminated surfaces are a potential repository for infectious bacteria [10]. Most studies investigating the contamination of mobile phones medical staff have examined medical doctors and nurses, and few studies have examined the contamination on mobile phones of dentists and dental hygienists. Singh et al. [11] investigated the bacterial contamination on mobile phones used by the medical staff of a dental college hospital in India. Potentially pathogenic microorganisms were detected on $34 \%$ of all mobile phones, and the bacterial contamination could be reduced by $87 \%$ after wiping the surface of the cell phone with alcohol. However, these previous studies detected microorganisms using traditional bacterial culture methods and identified the detected organisms using Gram staining and biochemical characteristics. These microbial identification methods have a disadvantage in that they cannot be used to analyze bacterial species that cannot be cultured. Therefore, here we investigated the bacterial diversity on mobile phones of dentists and dental hygienists through analysis of Illumina $16 \mathrm{~S}$ ribosomal DNA sequencing.

\section{MATERIALS AND METHODS}

\section{Sampling}

This study was carried out on 10 mobile phones that were randomly selected from five dentists and five dental hygienists working at Gangneung-Wonju National Univer- sity Dental Hospital. A sterilized cotton swab was soaked in sterile distilled water, and the entire surface of the mobile phone was rubbed to obtain bacteria. The swabs were then vortexed in $1 \mathrm{~mL}$ of sterile distilled water. A new sterile latex glove was used when sampling each mobile phone.

\section{Genomic DNA extraction}

To extract the DNA from the mobile phone swab samples, the PureLink Genomic DNA Mini Kit (Invitrogen, Waltham, MA, USA) was used according to the manufacturer's instructions. For Illumina sequencing analysis, the samples were divided into two groups: dentists (group A) and dental hygienists (group B), and the individual DNA samples from each group were mixed at the same ratio and analyzed together.

\section{Polymerase chain reaction amplification and Illumina sequencing}

The extracted DNA samples were analyzed using previously reported experimental methods [12,13]. The V3-V4 region of the 16S rRNA gene was amplified by polymerase chain reaction using the bacterial universal primer pair (341F-5'-TCGTCGGCAGCGTCAGATGTGTATAAGAGACAGCCTACGGGNGGCWGCAG-3', 805R-5'-GTCTCGTGGGCTCGG-AGATGTGTATAAGAGACAGGACTACHVGGGTAT CTAATCC- $3^{\prime}$ ). Next, a limited cycle amplification step was performed to add multiplexing indices and Illumina sequencing adapters. Then, the library was sequenced on the MiSeq platform. All subsequent analyses were performed with the BIOiPLUG pipeline, a commercially available ChunLab bioinformatics cloud platform for microbiome research.

\section{Pathogenic bacteria analysis}

The bacteria identified by the Illumina MiSeq analysis were used as queries in a search of the PubMed database for case reports related to pathogenicity over the last 10 years [14]. If one of species belonging to the identified genus was reported to be pathogenic, the genus was considered to be pathogenic. 


\section{RESULTS}

\section{Bacterial diversity in the mobile phone samples}

To determine how many types of microorganisms are present on mobile phones of dentists and dental hygienists in dental hospitals, the five DNA samples belonging to each of the two groups were mixed and subjected to Illumina sequencing. On the group A, a total of 54,710 sequence reads were obtained, and 84 genera were detected. On the group B, a total of 48,810 sequence read were obtained, and 101 genera were detected (Supplementary Table 1). Fig. 1 shows the species richness of each group. Fig. 2 and 3 show the bacterial diversity on the dentists' and dental hygienists' mobile phones. In group A, Pseudomonas spp. (52.52\%), Janthinobacterium spp. (14.21\%), Enterococcus spp. (9.66\%), Stenotrophomonas spp. (5.68\%), Streptococcus spp. (4.29\%), and Acinetobacter spp. (3.15\%) were detected. In group B, Enterococcus spp. (32.02\%), Pseudomonas spp. (23.76\%), Streptococcus spp. (22.44\%), Lactobacillus spp. (8.77\%), Janthinobacterium spp. (3.54\%), Acinetobacter spp. (1.7\%), and Stenotrophomonas spp. (1.22\%) were detected (Fig. 4).

\section{Pathogenic bacteria}

In group A, 32 of the 84 detected genera (38.09\%) include pathogenic species. Pseudomonas were frequently detected, and other pathogenic genera, such as Enterococcus spp., Stenotrophomonas spp., Acinetobacter spp., Morganella spp., Corynebacterium spp., Propionibacterium spp., Neisseria spp., Staphylococcus spp., Haemophilus spp., and Escherichia spp., were detected. Moreover, genera associated with oral diseases, such as Streptococcus spp., Prevotella spp., Actinomyces spp., Porphyromonas spp., and Fusobacterium spp., were also detected.

In group B, 26 of 101 genera (25.74\%) include pathogenic species, and Enterococcus spp., Pseudomonas spp., Acinetobacter spp., Stenotrophomonas spp., Escherichia spp., Bacillus spp., Corynebacterium spp., Staphylococcus spp., and Enterococcus spp., were detected. Moreover, bacterial genera associated with oral diseases, such as Streptococcus spp., Prevotella spp., Aggregatibacter spp., Porphyromonas spp., Actinomyces spp., and Fusobacterium spp., were also detected.

\section{DISCUSSION}

The possibility of infectious pathogens spreading through mobile phone use in hospitals was previously studied by Ulger et al. [8]. In the study, several of the identified bacterial species were important antibiotic-resistant pathogens. Most of the studies on the bacterial contamination of mobile phones of medical staff have been limited to doctors and nurses, and there are few studies on the contamination of mobile phones used by dentists. In addition, since previous studies used bacterial culture methods, there is a possibility that the contamination risk associated with mobile phone use in medical institutions may have been underestimated. In this study, Illumina sequencing was performed to overcome this limitation, as this method can detect all the bacteria present, including those that cannot be cultured in the laboratory.

In this study, most of the bacteria isolated from mobile phones of dentists and dental hygienists were microorganisms that live on the skin or in the environment, and although many were potentially pathogenic, most were of low toxicity. Therefore, it is difficult to conclude that these bacteria can directly cause infections in patients. However, contaminated mobile phones are likely to be mediate the transfer of pathogenic bacteria, as some of the bacteria detected on the cell phones were oral microorganisms originating from patients and pathogenic bacteria that can cause opportunistic infections.

Singh et al. [11] investigated the bacterial contamination on mobile phones of dentists at a dental college hospital in India. The study showed that coagulase-negative Staphylococcus spp. and Staphylococcus aureus were the most commonly found bacteria on mobile phones, and pathogens such as Bacillus spp., Acinetobacter spp., Pseudomonas spp., and Micrococci spp., and that Staphylococcus citreus and Diphtheroids as well as non-methicillinresistant $S$. aureus and vancomycin-resistant Enterococci spp. were also detected. However, their study differed from ours as the bacteria were identified using general culture methods. In our study, more opportunistic pathogens, 
such as Pseudomonas spp. and Janthinobacterium spp., were isolated from the dentists' mobile phones than from the hygienists' phones. In addition, Enterococcus spp. and

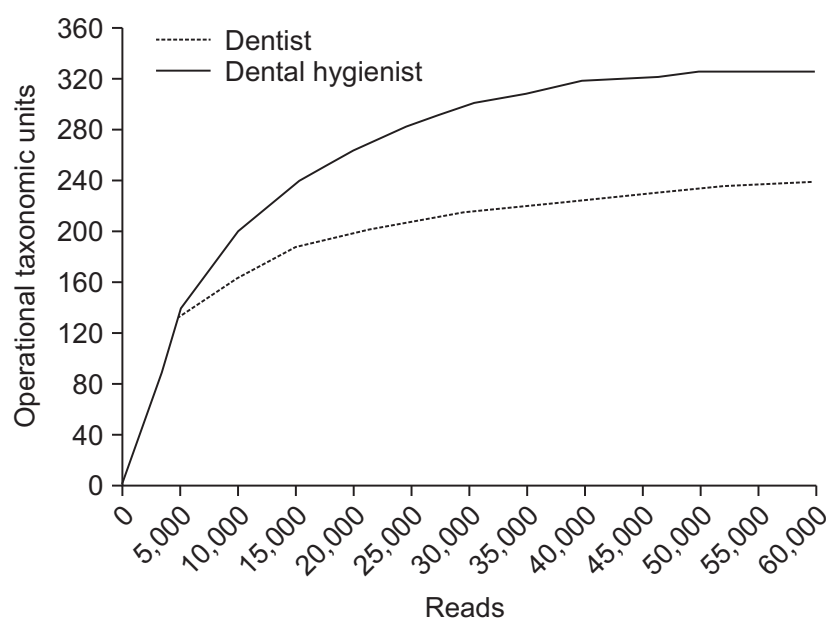

Fig. 1. Rarefaction curves of the bacterial communities in mobile phone samples.
Stenotrophomonas spp., which are known to cause serious nosocomial infections, and Streptococcus spp., which is associated with oral diseases, were also detected. Furthermore, Acinetobacter, a genus of opportunistic pathogens that are known to be multidrug resistant, was also detected. The types of microorganisms isolated from the dental hygienists' mobile phones were similar to those isolated from the dentists' mobile phones.

In our study, various opportunistic pathogens were detected. In particular, in both groups, Corynebacterium spp., which includes the causative agent of the respiratory infection diphtheria, Enterococcus spp., which are known to be multidrug resistant, and Escherichia spp., a genus of enteric bacteria, were detected. On the dentists' mobile phones, Haemophilus spp., which can cause a deadly respiratory infection, and Neisseria spp., which can cause septicemia and gonorrhea, were detected. In addition, Streptococcus spp., Actinomyces spp., Porphyromonas

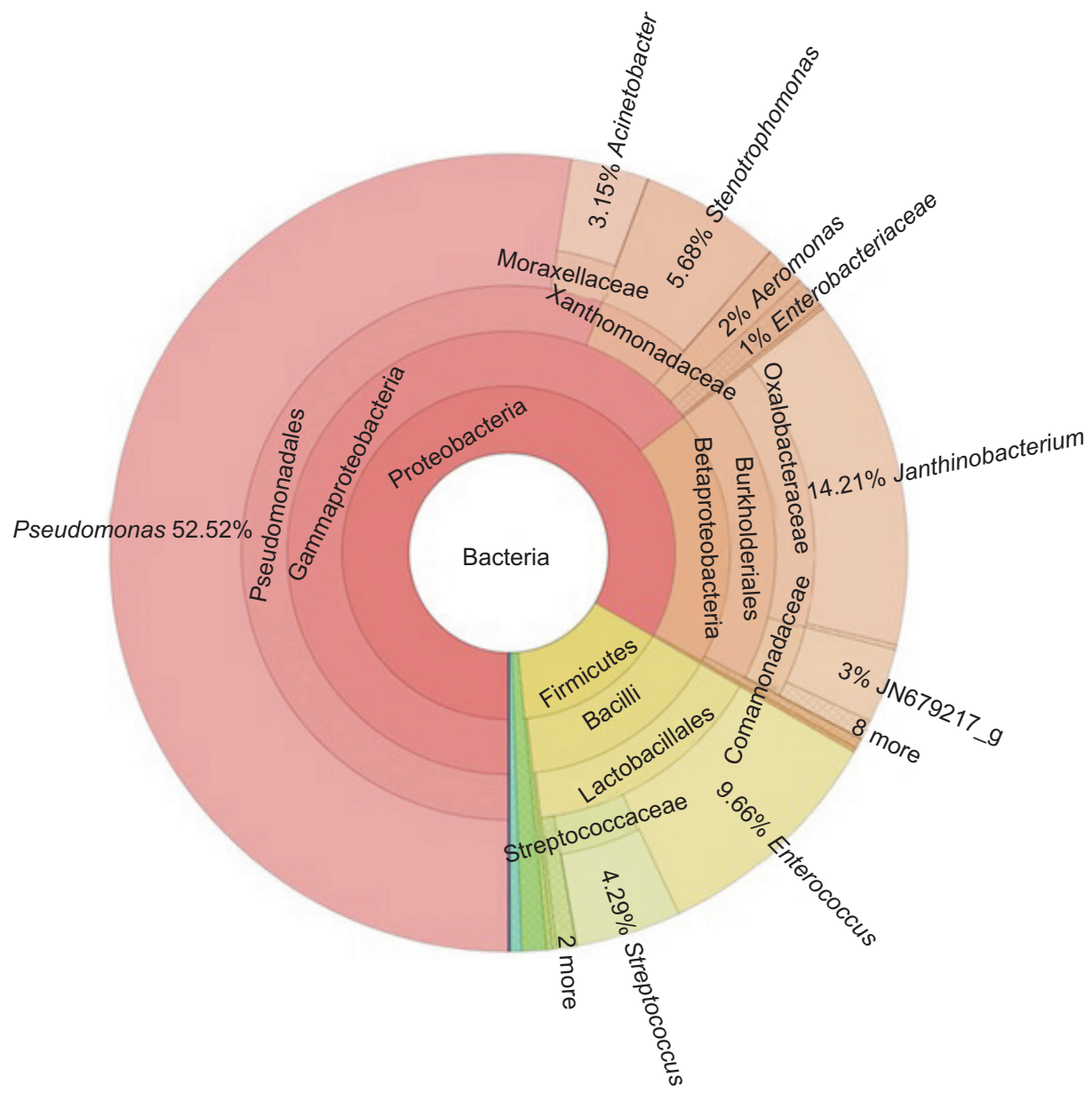

Fig. 2. Diversity of the bacterial communities present in mobile phone samples from dentists (group A). 


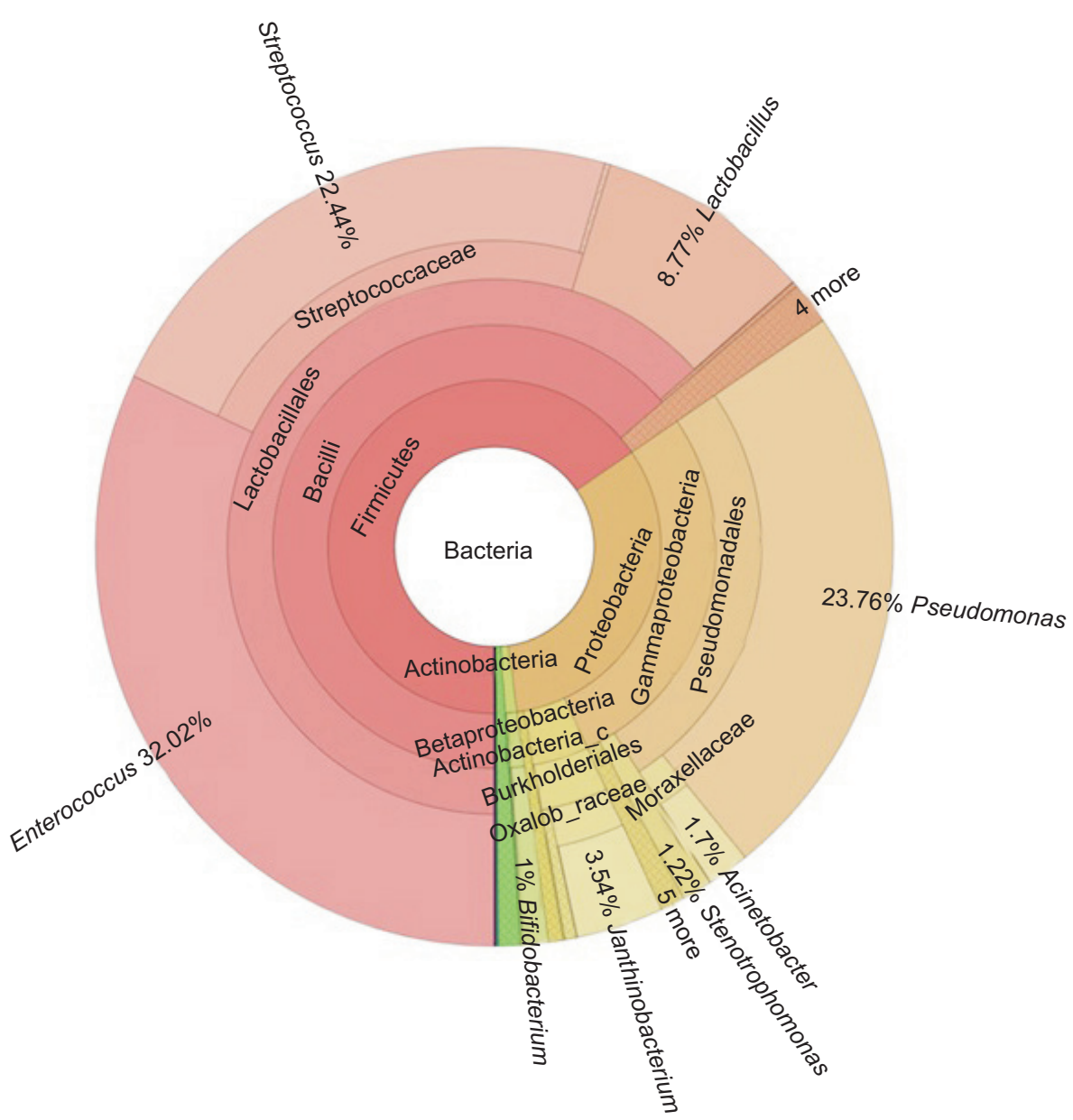

Fig. 3. Diversity of the bacterial communities present in mobile phone samples from dental hygienists (group B).

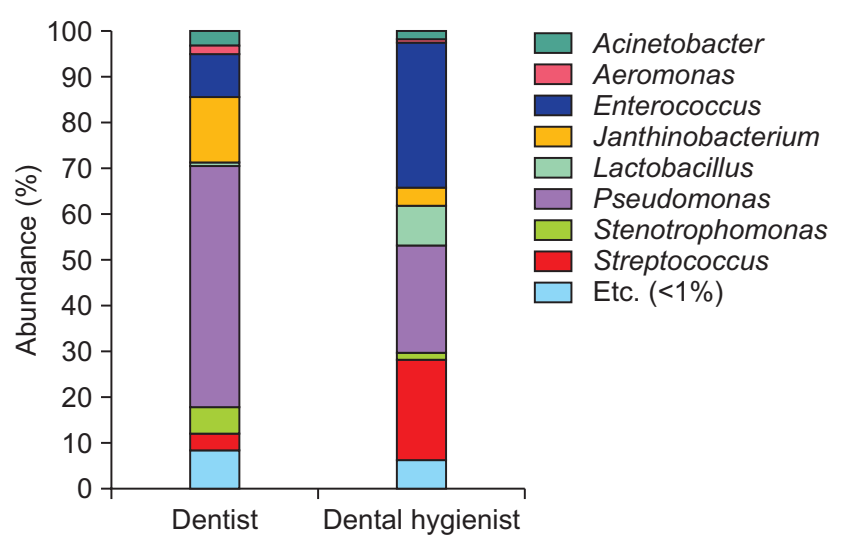

Fig. 4. Relative abundances of the bacterial genera detected in samples from mobile phones of dentists and dental hygienists.

spp., and Fusobacterium spp., which are known to be associated with various oral diseases, were detected in both groups. The intestinal enteric microorganism, Escherichia spp., is believed to have been transferred to mobile phones by dentists and dental hygienists who did not wash their hands properly after using the bathroom. In addition, the oral bacteria are thought to have been transferred to the phones by the hands of the dentists and dental hygienists after patient treatment. In some studies, it was reported that a doctor's mobile phones are more likely to be infected with pathogenic bacteria than a nurse's phone $[1,6,15]$. In our study, the percentages of pathogenic bacteria detected on the dentists' and dental hygienists' phones were similar. However, some bacteria, which are known to be pathogenic in humans, such as Haemophilus spp. and Neisseria spp., were only detected on the dentists' mobile phones.

This study shows that mobile phones used by dentists and dental hygienists can be contaminated by infectious pathogens. Our study was the first to analyze the contamination on mobile phones used by dentists and dental hygienists by Illumina sequencing. The clinical significance and propagation patterns of bacteria detected on mobile 
phones require further research and discussion. Our data demonstrate the need to set hygiene standards and pay careful attention to the possibility of pathogen dispersal by the dental staff in close contact with patients.

\section{CONFLICTS OF INTEREST}

The authors declare that they have no competing interests.

\section{ORCID}

\author{
So Yeon Lee \\ https://orcid.org/0000-0002-6645-6831 \\ Si Young Lee \\ https://orcid.org/0000-0001-8826-1413
}

\section{REFERENCES}

1. Brady RR, Verran J, Damani NN, Gibb AP. Review of mobile communication devices as potential reservoirs of nosocomial pathogens. J Hosp Infect 2009;71:295-300. doi: 10.1016/j.jhin.2008.12.009.

2. Ramesh J, Carter AO, Campbell MH, Gibbons N, Powlett C, Moseley H Sr, Lewis D, Carter T. Use of mobile phones by medical staff at Queen Elizabeth Hospital, Barbados: evidence for both benefit and harm. J Hosp Infect 2008;70:160-165. doi: 10.1016/j.jhin.2008.06.007.

3. Soto RG, Chu LF, Goldman JM, Rampil IJ, Ruskin KJ. Communication in critical care environments: mobile telephones improve patient care. Anesth Analg 2006;102:535541. doi: 10.1213/01.ane.0000194506.79408.79.

4. Ducel G, Fabry J, Nicolle L. Prevention of hospital-acquired infections: a practical guide. 2nd ed. Geneva: World Health Organization; 2002.

5. Singh D, Kaur H, Gardner WG, Treen LB. Bacterial contam- ination of hospital pagers. Infect Control Hosp Epidemiol 2002;23:274-276. doi: 10.1086/502048.

6. Braddy CM, Blair JE. Colonization of personal digital assistants used in a health care setting. Am J Infect Control 2005;33:230-232. doi: 10.1016/j.ajic.2005.03.003.

7. Karabay O, Kocoglu E, Tahtaci M. The role of mobile phones in the spread of bacteria associated with nosocomial infections. J Infect Dev Ctries 2007;1:72-73.

8. Ulger F, Esen S, Dilek A, Yanik K, Gunaydin M, Leblebicioglu $\mathrm{H}$. Are we aware how contaminated our mobile phones with nosocomial pathogens? Ann Clin Microbiol Antimicrob 2009;8:7. doi: 10.1186/1476-0711-8-7.

9. Neff JH, Rosenthal SL. A possible means of inadvertent transmission of infection to dental patients. J Dent Res 1957;36:932-934. doi: 10.1177/00220345570360061901.

10. Murray JP, Slack GL. Some sources of bacterial contamination in everyday dental practice. Br Dent J 1957;134:172174.

11. Singh S, Acharya S, Bhat M, Rao SK, Pentapati KC. Mobile phone hygiene: potential risks posed by use in the clinics of an Indian dental school. J Dent Educ 2010;74:11531158.

12. Lee SY, Lee SY. Assessment of bacterial contamination of lipstick using pyrosequencing. J Cosmet Sci 2017;68:245252.

13. Kim JY, Kim EM, Yi MH, Lee J, Lee S, Hwang Y, Yong D, Sohn WM, Yong TS. Intestinal fluke Metagonimus yokogawai infection increases probiotic Lactobacillus in mouse cecum. Exp Parasitol 2018;193:45-50. doi: 10.1016/ j.exppara.2018.08.002.

14. Costa D, Mercier A, Gravouil K, Lesobre J, Delafont V, Bousseau A, Verdon J, Imbert C. Pyrosequencing analysis of bacterial diversity in dental unit waterlines. Water Res 2015;81:223-231. doi: 10.1016/j.watres.2015.05.065.

15. Goldblatt JG, Krief I, Klonsky T, Haller D, Milloul V, Sixsmith DM, Srugo I, Potasman I. Use of cellular telephones and transmission of pathogens by medical staff in New York and Israel. Infect Control Hosp Epidemiol 2007;28:500-503. doi: 10.1086/513446. 
Supplementary Table 1. List of all the genera identified in the samples from mobile phones of dentists and dental hygienists using Illumina MiSeq

\begin{tabular}{|c|c|c|c|c|}
\hline \multirow{2}{*}{ Phylum } & \multirow{2}{*}{ Class } & \multirow{2}{*}{ Genus } & \multicolumn{2}{|c|}{ Count } \\
\hline & & & Dentist & Dental hygienist \\
\hline \multirow[t]{14}{*}{ Actinobacteria } & Actinobacteria_c & Actinomyces & 12 & 2 \\
\hline & & Arcanobacterium & 1 & - \\
\hline & & Bifidobacterium & 159 & 486 \\
\hline & & Brevibacterium & - & 4 \\
\hline & & Calidifontibacter & 4 & - \\
\hline & & Cellulomonas & - & 2 \\
\hline & & Corynebacterium & 112 & 13 \\
\hline & & Gardnerella & 12 & - \\
\hline & & Micrococcus & 14 & - \\
\hline & & Nocardioides & 4 & - \\
\hline & & Phycicoccus & 36 & - \\
\hline & & Propionibacterium & 103 & 14 \\
\hline & & Rhodococcus & 3 & - \\
\hline & & Rothia & 42 & 2 \\
\hline \multirow[t]{12}{*}{ Bacteroidetes } & Bacteroidia & Alistipes & 21 & 69 \\
\hline & & Alloprevotella & 31 & 14 \\
\hline & & Bacteroides & - & 14 \\
\hline & & Parabacteroides & - & 7 \\
\hline & & Porphyromonas & 68 & 2 \\
\hline & & Prevotella & 58 & 63 \\
\hline & Flavobacteria & Capnocytophaga & 6 & - \\
\hline & & Flavobacterium & 1 & - \\
\hline & & Litoribaculum & 1 & - \\
\hline & & Tenacibaculum & 1 & - \\
\hline & Sphingobacteriia & Ferruginibacter & - & 3 \\
\hline & & Sphingobacterium & 8 & - \\
\hline Deferribacteres & Deferribacteres_c & Mucispirillum & - & 9 \\
\hline Deinococcus-Thermus & Deinococci & Meiothermus & 3 & - \\
\hline \multirow[t]{22}{*}{ Firmicutes } & Bacilli & Alkalibacterium & - & 1 \\
\hline & & Bacillus & - & 41 \\
\hline & & Enterococcus & 5,284 & 15,687 \\
\hline & & Gemella & 20 & 2 \\
\hline & & Granulicatella & 6 & 4 \\
\hline & & Lactobacillus & 429 & 4,295 \\
\hline & & Lactococcus & 1 & 112 \\
\hline & & Paenibacillus & 12 & - \\
\hline & & Staphylococcus & 55 & 3 \\
\hline & & Streptococcus & 2,345 & 10,995 \\
\hline & Clostridia & Acidaminobacter & 19 & 181 \\
\hline & & Alkalibacter & - & 25 \\
\hline & & Alkaliphilus & - & 39 \\
\hline & & Anaerofilum & - & 8 \\
\hline & & Anaerotruncus & - & 3 \\
\hline & & Anoxynatronum & - & 7 \\
\hline & & Butyricicoccus & - & 8 \\
\hline & & Clostridium & 15 & 5 \\
\hline & & Dehalobacter & - & 5 \\
\hline & & Dorea & - & 3 \\
\hline & & Eisenbergiella & 2 & 26 \\
\hline & & Ercella & - & 1 \\
\hline
\end{tabular}


Supplementary Table 1. Continued

\begin{tabular}{|c|c|c|c|c|}
\hline \multirow{2}{*}{ Phylum } & \multirow{2}{*}{ Class } & \multirow{2}{*}{ Genus } & \multicolumn{2}{|c|}{ Count } \\
\hline & & & Dentist & Dental hygienist \\
\hline & & Eubacterium & 7 & 9 \\
\hline & & Faecalibacterium & - & 11 \\
\hline & & Lachnospira & - & 2 \\
\hline & & Moryella & 10 & - \\
\hline & & Oscillibacter & - & 6 \\
\hline & & Pseudoflavonifractor & - & 4 \\
\hline & & Ruminococcus & - & 1 \\
\hline & & Ruminococcus_g4 & - & 2 \\
\hline & & Subdoligranulum & - & 2 \\
\hline & & Tindallia & - & 8 \\
\hline & Erysipelotrichi & Bulleidia & 11 & - \\
\hline & Negativicutes & Pelosinus & 26 & 10 \\
\hline & & Phascolarctobacterium & - & 5 \\
\hline & & Selenomonas & 2 & - \\
\hline & & Veillonella & - & 11 \\
\hline & Tissierellia & Anaerococcus & 7 & - \\
\hline & & Dethiosulfatibacter & - & 118 \\
\hline & & Tissierella & - & 181 \\
\hline \multirow[t]{2}{*}{ Fusobacteria } & Fusobacteria_c & Fusobacterium & 32 & 1 \\
\hline & & Leptotrichia & 1 & - \\
\hline \multirow[t]{30}{*}{ Proteobacteria } & Alphaproteobacteria & Acetobacter & - & 2 \\
\hline & & Altererythrobacter & 16 & 3 \\
\hline & & Bartonella & - & 12 \\
\hline & & Bradyrhizobium & - & 3 \\
\hline & & Brevundimonas & 12 & - \\
\hline & & Caulobacter & 4 & - \\
\hline & & Erythrobacter & - & 1 \\
\hline & & Methylobacterium & 12 & 18 \\
\hline & & Methyloceanibacter & - & 1 \\
\hline & & Novosphingobium & - & 5 \\
\hline & & Shinella & 3 & - \\
\hline & & Sphingobium & 13 & - \\
\hline & & Sphingomonas & 30 & 14 \\
\hline & Betaproteobacteria & Acidovorax & 49 & 4 \\
\hline & & Aquabacterium & - & 2 \\
\hline & & Comamonas & 26 & 5 \\
\hline & & Curvibacter & 9 & - \\
\hline & & Dechloromonas & 134 & 28 \\
\hline & & Delftia & 197 & 23 \\
\hline & & Herbaspirillum & 8 & 3 \\
\hline & & Janthinobacterium & 7,774 & 1,736 \\
\hline & & Kinneretia & 8 & - \\
\hline & & Lautropia & 5 & - \\
\hline & & Massilia & 13 & 1 \\
\hline & & Neisseria & 66 & - \\
\hline & & Parasutterella & - & 3 \\
\hline & & Paucibacter & 145 & 23 \\
\hline & & Rhizobacter & - & 4 \\
\hline & & Schlegelella & 1 & - \\
\hline & & Undibacterium & 87 & 16 \\
\hline
\end{tabular}


Supplementary Table 1. Continued

\begin{tabular}{|c|c|c|c|c|}
\hline \multirow{2}{*}{ Phylum } & \multirow{2}{*}{ Class } & \multirow{2}{*}{ Genus } & \multicolumn{2}{|c|}{ Count } \\
\hline & & & Dentist & Dental hygienis \\
\hline & Deltaproteobacteria & Desulfonatronum & 8 & 230 \\
\hline & Epsilonproteobacteria & Campylobacter & - & 1 \\
\hline & & Sulfurimonas & - & 1 \\
\hline & & Sulfurovum & 1 & 1 \\
\hline & Gammaproteobacteria & Acinetobacter & 1,723 & 834 \\
\hline & & Aeromonas & 891 & 246 \\
\hline & & Aggregatibacter & - & 5 \\
\hline & & Buttiauxella & 18 & 11 \\
\hline & & Cedecea & 111 & 19 \\
\hline & & Citrobacter & 51 & 34 \\
\hline & & Enhydrobacter & 16 & 13 \\
\hline & & Enterobacter & 6 & 1 \\
\hline & & Escherichia & 47 & 65 \\
\hline & & Haemophilus & 12 & - \\
\hline & & Halomonas & 86 & 12 \\
\hline & & Lelliottia & 6 & - \\
\hline & & Morganella & 372 & 61 \\
\hline & & Pantoea & 1 & - \\
\hline & & Providencia & - & 2 \\
\hline & & Pseudomonas & 28,736 & 11,641 \\
\hline & & Rahnella & 2 & 7 \\
\hline & & Serratia & 6 & 10 \\
\hline & & Siccibacter & 14 & - \\
\hline & & Stenotrophomonas & 3,106 & 599 \\
\hline & & Vibrio & - & 6 \\
\hline & & Xanthomonas & 12 & - \\
\hline & & Yersinia & 71 & 3 \\
\hline Synergistetes & Synergistia & Aminivibrio & - & 12 \\
\hline Tenericutes & Mollicutes & Acholeplasma_g2 & - & 3 \\
\hline Verrucomicrobia & Verrucomicrobiae & Akkermansia & - & 8 \\
\hline Unknown & & & 1,818 & 552 \\
\hline Total & & & 54,710 & 48,810 \\
\hline
\end{tabular}

